\title{
INFLUENCE OF SORET EFFECT ON MHD MIXED CONVECTION FLOW OF VISCO-ELASTIC FLUID PAST A VERTICAL SURFACE WITH HALL EFFECT
}

\author{
A.K. JHA \\ Department of Mathematics, Jecrccun Iversity College \\ Sitapura, Jaipur-302022 INDIA \\ E-mail: itsabhay@rediffmail.com \\ K. CHOUDHARY* \\ Department of Mathematics, Global Institute of Technology \\ Sitapura, Jaipur-302022, INDIA \\ E-mail: dr.kchoudhary@yahoo.com \\ A. SHARMA \\ Department of Mathematics, University of Rajasthan \\ Jaipur-302004, INDIA
}

\begin{abstract}
The present paper deals with the unsteady motion of an MHD free convection flow of an incompressible nonNewtonian viscoelastic fluid past an infinite vertical plate in the presence of a heat source and Soret effect. A parametric study illustrating the influence of various parameters on the temperature, velocity as well as on the skin-friction and rate of heat transfer is conducted. The results of the effect of the magnetic field, the parameter describing the non-Newtonian behavior, and the velocity of suction and injection on both the velocity and temperature distributions are examined and shown graphically.
\end{abstract}

Key words: thermal diffusion, Hall parameter, MHD, elastic parameter, Schmidt number, heat source.

\section{Introduction}

Magnetohdrodynamics (MHD) is currently undergoing a period of great extension and differentiation of subject matter. The interest in these new problems originates from their importance in liquid metals electrolytes and ionized gas. The MHD heat and mass transfer processes are of interest in power engineering, metallurgy astrophysics and geophysics. On account of their varied importance, these flows have been studied by several authors notable amongst them are Shercliff (1965), Ferraro and Plumbton (1966) and Cramer and Pai (1973). Elbashbeshy (1997) studied heat and mass transfer along a vertical plate in the presence of a magnetic field. Singh $(2001 ; 2003)$ analyzed an MHD free convection and mass transfer flow with a heat source and thermal diffusion combined with heat and mass transfer in an MHD free convection from a vertical surface was studied by Chein (2004). The effect of Hall current on the fluid flow with variable concentration has many application in MHD power generations, in several astrophysical and metrological studies as well as in flow of plasma through MHD generators. From the point of application, model studies on the Hall effect on free and forced convection flows have been made by several investigators. Katagiri (1969) studied the effects of Hall current on the magneto hydrodynamic boundary layer flow past a semi infinite vertical plate. Pop and Soundalgekar (1974) and Gupta (1975) investigated the

\footnotetext{
* To whom correspondence should be addressed
} 
effect of Hall current on the steady hydromagnetic flow in an incompressible viscous fluid. Hossain and Rashid (1987) discussed the effect of Hall current on a steady free convection flow along porous plate in the presence of foreign gases such as $\mathrm{H}_{2}, \mathrm{CO}_{2}$ and $\mathrm{NH}_{3}$ subjected to a transpiration velocity inversely proportional to a square root of time. Acharya (2001a; 2001b) analyzed the Hall effect with simultaneous thermal and mass diffusion on an unsteady hydromagnetic flow past a vertical plate. Assuming constant suction injection normal to the plate they solved the problem analytically. Abeldahab and Elbrbary (2001) discussed heat and mass transfer along a vertical plate under the combined buoyancy force effects of thermal and species diffusion in the presence of a transversely applied magnetic field and taking the Hall effect into account. The Hall effect on an MHD free convection flow of a viscous incompressible fluid past an vertical plate in porous medium with heat source/sink was discussed by Sharma et al. (2007).

Recently, researchers in engineering and scientific fields have shown great interest in the study of a non-Newtonian fluid due to its importance in industrial processes. Many authors have examined the flow, heat and mass transfer in non-Newtonian fluids of different types. Sarpakaya (1961) discussed the steady flow of a uniformly conducting non-Newtonian incompressible fluid between two parallel plates. The fluid considered is under the influence of a constant pressure gradient. Chaudhary and Jain (2006) analyzed the Hall effect on an MHD mixed convection flow of a viscoelastic fluid past an infinite vertical porous plate with mass transfer and radiation. Chaudhary and Jha (2008) discussed heat and mass transfer in an elasticoviscous fluid past an impulsively started infinite vertical plate with the Hall effect.

In all above studied, the thermal-diffusion effects are negligible. However, the thermal-diffusion effects, which are caused by the temperature gradient (called the Soret effect) is an interesting macroscopically physical phenomenon in fluid mechanics. Usually, in heat and mass transfer problems the variation of density with temperature gives rise to the considered buoyancy effect under natural convection and hence the temperature will influence the diffusion species. Alam et al. (2006) studied the Soret Dufour effect on a steady MHD combined free -forced convection and mass transfer flow past a semi-infinite vertical plate. Pal and Mondal (2011a; 2011b) examined the effect of Soret and Dufour on an MHD nonDarcy unsteady mixed convection heat and mass transfer over a stretching sheet.

\section{Mathematical formulation}

The constitutive equations for the rheological equation of state for an elastic-viscous fluid (Walters' liquid B') are

$$
\begin{aligned}
& p_{i k}=-p g_{i k}+p_{i k}, \\
& p_{i k}=2 \int_{-\infty}^{l} \psi\left(t-t^{\prime}\right) e_{i k}^{(l)}\left(t^{\prime}\right) d t^{\prime}, \\
& \psi\left(t-t^{\prime}\right)=\int_{0}^{\infty} \frac{N(\tau)}{\tau} e^{-\left(t-t^{\prime}\right) \tau} d \tau .
\end{aligned}
$$

$N(\tau)$ is the distribution function of relaxation times, in the above equation $p_{i k}$ and $p$ are, respectively, the stress tensor an arbitrary isotropic pressure, $g_{i k}$ is the metric tensor of a fixed co-ordinate system $x_{i}$ and $e_{i k}^{(l)}$ is the rate of strain tensor. It was shown by Walters (1964) that Eq.(2.2) can be put in the following generalized form which is valid for all types of motion and stress

$$
p^{i k}(x, t)=2 \int_{-\infty}^{l} \psi\left(t-t^{\prime}\right) \frac{\partial x^{\prime}}{\partial x^{m}} \frac{\partial x^{k}}{\partial x^{\prime r}} e^{(l) m t}\left(x^{\prime} t^{\prime}\right) d t^{\prime}
$$


where $x^{1}$ is the position at time $t$ ' of the element that is instantaneously at the point $x$ ' at time " $t$ ". The fluid with equation of state (2.1) and (2.4) has been designated as liquid B' in the case of liquids with short memories, i.e., short relaxation times, the above equation of state can be written in the following simplified form

$$
p^{i k}(x, t)=2 \eta_{0} e^{(l) i k}-2 k_{0} \frac{\partial e^{(I) i k}}{\partial t}
$$

in which $\eta_{0}=\int_{0}^{\infty} N(\tau) d \tau$ is limiting viscosity at small rate shear,

$k_{0}=\int_{0}^{\infty} \tau N(\tau) d \tau$ and $\frac{\partial}{\partial t}$ denotes the convected time derivative.

We consider the unsteady flow of a viscous incompressible and electrically conducting elastoviscous fluid with oscillating temperature and concentration, the flow occurs along the $x$-axis which is taken to be along the plate and the $y$-axis is taken normal to it. The plate starts moving in its own plane with uniform velocity $v_{0}$ (a constant velocity). A uniform magnetic field is applied normal to the plate with constant suction. The equations governing the flow of the fluid are as follows

Equation of continuity

$$
\nabla \cdot V=0
$$

Momentum equation

$$
\frac{\partial V}{\partial t}+(V \cdot \nabla) V=-\frac{1}{\rho} \nabla P+\nabla \cdot p_{i j}+g \beta\left(T-T_{\infty}\right)+g \beta^{*}\left(C-C_{\infty}\right)+\frac{1}{\rho}(J \times B) .
$$

Generalized Ohm's law

$$
J=\sigma(E+V \times B)-\frac{\sigma}{e n_{e}}\left(J \times B-\nabla p_{e}\right)
$$

where $v=(u, v, w)$ is the velocity field, $P$ is the pressure field, $g$ is acceleration due to gravity, $\beta$ the volumetric coefficient of thermal expansion, $\beta^{*}$ the volumetric coefficient of volume expansion for mass transfer $\rho$ the density of the fluid, $J$ is the current density, $M$ is the magnetic field, $E$ is the electric field and $p_{i j}$ is the stress tensor. The effect of Hall current induces a force which causes a cross flow in the $z$-direction, therefore the flow becomes three dimensional. It is assumed that there is no applied or polarization voltage so that $E=0$ and the induced field $B=\left(0, B_{0}, 0\right)$, where $B_{0}$ is the applied magnetic field parallel to the $y$-axis. This assumption is justified when the magnetic Reynolds number is very small.

The generalized Ohm's law including Hall current is given in the form

$$
J+\frac{\omega_{e} \tau_{e}}{B_{0}}(\boldsymbol{J} \times \boldsymbol{B})=\sigma\left(\boldsymbol{J} \times \boldsymbol{B}+\frac{1}{e n_{e}} \nabla p_{e}+\boldsymbol{E}\right)
$$

where $\omega_{e}$ is the electron frequency, $\tau_{e}$ is the electron collision time, $J$ is the electron conductivity, $p_{e}$ is the electron pressure and $n_{e}$ is the number density of electron. For weakly ionized gases the thermoelectric pressure and ion slip are considered negligible. Then Eq.(2.9) reduces to 


$$
\begin{aligned}
& J_{X}=\frac{\sigma B_{0}}{\left(1+m^{2}\right)}(m u-w), \\
& J_{Z}=\frac{\sigma B_{0}}{\left(1+m^{2}\right)}(u+m w)
\end{aligned}
$$

where $u, v$ and $w$ are $x, y$ and $z$ components of velocity vector, respectively, is the Hall parameter defined by $m=\omega_{e} \tau_{e}$.

As the plate is of infinite length all the physical variables in this problem are functions of $y$ and $t$. Boussinesq approximation equations governing the flows are as follows

equation of continuity

$$
\frac{\partial v}{\partial y}=0
$$

$V=-v_{0}$ where $v_{0}$ is the constant suction velocity;

momentum equation

$$
\begin{aligned}
& \frac{\partial u}{\partial t}+v \frac{\partial u}{\partial y}=v \frac{\partial^{2} u}{\partial y^{2}}-k_{0} \frac{\partial^{3} u}{\partial y^{2} \partial t}-\frac{\sigma B_{0}^{2}(u+m w)}{\rho\left(1+m^{2}\right)}+g \beta\left(T-T_{\infty}\right)+g \beta^{*}\left(C-C_{\infty}\right), \\
& \frac{\partial w}{\partial t}+v \frac{\partial w}{\partial y}=v \frac{\partial^{2} w}{\partial y^{2}}-k_{0} \frac{\partial^{3} w}{\partial y^{2} \partial t}-\frac{\sigma B_{0}^{2}(w-m u)}{\rho\left(1+m^{2}\right)}
\end{aligned}
$$

energy equation

$$
\frac{\partial T}{\partial t}+v \frac{\partial T}{\partial y}=\frac{k}{\rho c_{p}} \frac{\partial^{2} T}{\partial y^{2}}+S\left(T-T_{\infty}\right),
$$

concentration equation

$$
\frac{\partial C}{\partial t}+v \frac{\partial C}{\partial y}=D \frac{\partial^{2} C}{\partial y^{2}}+D_{T} \frac{\partial^{2}\left(T-T_{\infty}\right)}{\partial y^{2}}
$$

where $v$ is the kinematic viscosity, $k_{0}$ is the elastic parameter, $T$ is the temperature, $C$ is the concentration, $k$ is the thermal conductivity, $c_{p}$ is the specific heat of the fluid and $g$ is the acceleration due to gravity, $s$ is the heat source parameter and $D_{T}$ is the Soret- Duffer effect coefficient. In Eq.(2.15) the terms due to viscous dissipation are neglected and in Eq.(2.16) the term due to the chemical reaction is assumed to be absent.

The initial boundary conditions are

$$
t \leq 0, \quad u(y, t)=w(y, t)=0, \quad T=0, \quad C=0 \quad \text { for } \quad \text { all } \quad y,
$$




$$
t>0\left\{\begin{array}{l}
u(0, t)=U_{0}, w(0, t)=0, \quad T=T_{\infty}+e^{i \omega t}\left(T_{w}-T_{\infty}\right) \\
C(0, t)=C_{\infty}+e^{i \omega t}\left(C_{w}-C_{\infty}\right), \quad \text { at } \quad y=0, \\
u(\infty, t)=w(\infty, t), \quad T(\infty, t)=C(\infty, t)=0 \quad \text { as } y \rightarrow \infty
\end{array}\right.
$$

where $\omega$ is the frequency of oscillation, and subscripts $w$ and $\infty$ denote the physical quantities at the plate and in the free stream, respectively.

We introduce the following non-dimensional quantities as follows

$$
\begin{aligned}
& \eta=\frac{V_{0} y}{v}, \quad \bar{t}=\frac{V_{0}^{2} t}{4 v}, \quad \bar{u}=\frac{u}{U_{O}}, \quad \bar{w}=\frac{w}{U_{0}}, \\
& \bar{\theta}=\frac{\left(T-T_{\infty}\right)}{\left(T_{w}-T_{\infty}\right)}, \quad \bar{C}=\frac{C-C_{\infty}}{C_{w}-C_{\infty}}, \\
& \Omega=\frac{4 \omega v}{V_{0}^{2}}, \quad \alpha=\frac{K_{0} V_{0}^{2}}{v^{2}}, \quad \operatorname{Pr}=\frac{v \rho C_{P}}{k}, \quad \mathrm{Sc}=\frac{v}{D}, \quad M=\frac{4 \sigma B_{0}{ }^{2} v \sigma}{\rho V_{0}^{2}}, \\
& \mathrm{Gr}=\frac{v \beta g\left(T_{W}^{\prime}-T_{\infty}^{\prime}\right)}{U_{0} V_{0}^{2}}, \quad \mathrm{Gm}=\frac{v \beta^{*} g\left(C_{W}^{\prime}-C_{\infty}^{\prime}\right)}{U_{0} V_{0}^{2}}, \\
& S=\frac{4 S v}{V_{0}^{2}}, \quad \mathrm{~S}_{0}=\frac{D_{T} a}{v b} .
\end{aligned}
$$
the bars)

Equations (2.13) to (2.16) transform to the following non-dimensional forms, respectively (dropping

$$
\begin{aligned}
& \frac{\partial u}{\partial t}-4 \frac{\partial u}{\partial \eta}=4 \frac{\partial^{2} u}{\partial \eta^{2}}-\alpha \frac{\partial^{2} u}{\partial \eta^{2} \partial t}-\frac{M}{1+m^{2}}(m w+u)+G \theta+\mathrm{Gc} C \\
& \frac{\partial w}{\partial t}-4 \frac{\partial w}{\partial \eta}=4 \frac{\partial^{2} w}{\partial \eta^{2}}-\alpha \frac{\partial^{2} w}{\partial \eta^{2} \partial t}-\frac{M}{1+m^{2}}(w-m u) \\
& \frac{\partial \theta}{\partial t}-4 \frac{\partial \theta}{\partial \eta}=\frac{4}{\operatorname{Pr}} \frac{\partial^{2} \theta}{\partial \eta^{2}}-S \theta \\
& \frac{\partial C}{\partial t}-4 \frac{\partial C}{\partial \eta}=\frac{4}{\operatorname{Sc}} \frac{\partial^{2} C}{\partial \eta^{2}}+4 S_{0} \frac{\partial^{2} \theta}{\partial \eta^{2}} \\
& t \leq 0, \quad u(\eta, t)=w(\eta, t)=\theta(\eta, t)=C(\eta, t)=0 \forall \eta
\end{aligned}
$$




$$
t>0\left\{\begin{array}{l}
u(0, t)=1, \quad w(0, t)=0, \quad \theta(0, t)=e^{i \Omega t}, \\
C(0, t)=e^{i \omega t}, \quad \text { at } \quad \eta=0, \\
u(\infty, t)=w(\infty, t), \quad \theta(\infty, t)=C(\infty, t)=0 \quad \text { as } \quad \eta \rightarrow \infty .
\end{array}\right.
$$

\section{Solution}

Equations (2.19) and (2.20) can be combined using the complex variable

$$
\psi=u+i w
$$

Equations (2.19)-(2.20)

$$
\frac{\partial^{2} \psi}{\partial \eta^{2}}-\alpha \frac{\partial^{2} \psi}{\partial \eta^{2} \partial t}-\frac{1}{4} \frac{\partial \psi}{\partial t}+\frac{\partial \psi}{\partial \eta}-\frac{M(1-i m)}{4\left(1+m^{2}\right)} \psi=-\frac{G \theta}{4}-\frac{\mathrm{GcC}}{4}
$$

Using Eq.(3.1) we get the boundary condition

$$
\begin{aligned}
& \psi(0, t)=1, \quad \psi(\infty, t)=0, \quad C(0, t)=e^{i \Omega t}, \\
& \theta(0, t)=e^{i \omega t}, \quad \theta(\infty, t)=0, \quad C(\infty, t)=0 .
\end{aligned}
$$

Putting $\theta(\eta, t)=e^{i \Omega t} f(\eta)$ in Eq.(2.21), we get

$$
f^{\prime \prime}(\eta)+\operatorname{Pr} f^{\prime}(\eta)-\left(\frac{i \Omega \operatorname{Pr}}{4}-\frac{S \operatorname{Pr}}{4}\right) f(\eta)=0 .
$$

Equation (3.4) can be solved under the following boundary conditions,

$$
f(0)=1, \quad f(\infty)=0 .
$$

Hence the solution is

$$
\begin{aligned}
& f(\eta)=e^{-i A_{1} \eta}, \\
& \theta(\eta, t)=e^{i \Omega t-A_{1} \eta}, \\
& \theta(\eta, t)=\cos \left(\Omega t-x_{2} \eta\right) \cdot e^{-\frac{x_{I} \eta}{2}}, \\
& \theta(\eta, t)=\cos \left(\Omega t-\beta_{1} \frac{\eta}{2} \sin \frac{\alpha_{1}}{2}\right) \cdot e^{-\frac{\eta}{2}\left(\operatorname{Pr}+x_{2} \cos \frac{\alpha_{1}}{2}\right)},
\end{aligned}
$$


where

$$
\begin{aligned}
& x_{2}=\left[\left(\operatorname{Pr}^{2}-\operatorname{Pr} S\right)^{2}+\operatorname{Pr}^{2} \Omega^{2}\right]^{\frac{1}{4}} \sin \frac{\alpha_{1}}{2}, \\
& \beta_{1}=\left[\left(\operatorname{Pr}^{2}-\operatorname{Pr} S\right)^{2}+\operatorname{Pr}^{2} \Omega^{2}\right]^{\frac{1}{4}}, \\
& \alpha_{1}=\tan ^{-1}\left(\frac{\Omega}{\operatorname{Pr}}\right), \\
& x_{1}=\operatorname{Pr}+\left[\left(\operatorname{Pr}^{2}-\operatorname{Pr} S\right)^{2}+\operatorname{Pr}^{2} \Omega^{2}\right]^{\frac{1}{2}} \cos \frac{\alpha_{1}}{2} .
\end{aligned}
$$

Putting $C(\eta, t)=e^{i \Omega t} g(\eta)$ in Eq.(2.23), we have

$$
g^{\prime \prime}(\eta)+\operatorname{Sc}^{\prime}(\eta)-\frac{i \Omega \operatorname{Sc}}{4} g(\eta)=-S_{0} \operatorname{Sc} f^{\prime \prime}(\eta)
$$

Equation (3.8) can be solved under the boundary conditions

$$
g(0)=1, \quad g(\infty)=0 .
$$

Consequently, the solution is

$$
\begin{aligned}
& g(\eta)=A_{3} e^{-A_{2} \eta}+A_{4} e^{-A_{1} \eta}, \\
& C(\eta, t)=e^{i \Omega t}\left(A_{3} e^{-A_{2} \eta}+A_{4} e^{-A_{l} \eta}\right) .
\end{aligned}
$$

Separating Eq.(3.9) the real and imaginary parts and considering only the real part, we get

$$
\begin{aligned}
& C_{r}=e^{-x_{3} \eta}\left[x_{9} \cos \left(\Omega t-x_{4} \eta\right)-x_{8} \sin \left(\Omega t-x_{4} \eta\right)\right]+ \\
& +e^{-x_{1} \eta}\left[x_{7} \cos \left(\Omega t-x_{2} \eta\right)-x_{8} \sin \left(\Omega t-x_{2} \eta\right)\right] .
\end{aligned}
$$

Separating the real and imaginary part

$$
\begin{aligned}
& A_{4}=x_{7}+i x_{8}, \\
& x_{9}=1-x_{7}, \quad A_{3}=1-A_{4}, \\
& x_{3}=\mathrm{Sc}+\alpha_{2}^{\frac{1}{2}} \cos \frac{\beta_{2}}{2}, \quad x_{4}=\alpha_{2}^{\frac{1}{2}} \sin \frac{\beta_{2}}{2}
\end{aligned}
$$


$\quad \quad \alpha_{2}=\operatorname{Sc}\left(\mathrm{Sc}^{2}+\Omega^{2}\right)^{\frac{1}{2}}, \quad \beta_{2}=\tan \left(\frac{\Omega}{\mathrm{Sc}}\right)$

Also substituting $\psi=e^{i \Omega t} F(\eta)$ in Eq.(3.2), we arrive at

$$
F^{\prime \prime}(\eta)(1-i \alpha)+F(\eta)-F(\eta)\left[\frac{i \Omega}{4}+\frac{M}{1+m^{2}}-\frac{i M m}{1+m^{2}}\right]=-\frac{1}{4} \operatorname{Gr} f(\eta)-\frac{1}{4} \operatorname{Gcg}(\eta)
$$

The equation can be solved under the boundary conditions

$$
F(0)=e^{-i \Omega t}, \quad F(\infty)=0 .
$$

Therefore the solution is

$$
\begin{aligned}
& F(\eta)=A_{15} e^{-A_{7} \eta}+A_{11} e^{-A_{1} \eta}+A_{14} e^{-A_{2} \eta}, \\
& \psi(\eta, t)=e^{i \Omega t}\left(A_{15} e^{-A_{7} \eta}+A_{11} e^{-A_{1} \eta}+A_{14} e^{-A_{2} \eta}\right)
\end{aligned}
$$

where

$$
x_{2}=\left[\left(\operatorname{Pr}^{2}-\operatorname{Pr} S\right)^{2}+\operatorname{Pr}^{2} \Omega^{2}\right]^{\frac{1}{4}} \sin \frac{\alpha_{1}}{2}, \quad \beta_{1}=\left[\left(\operatorname{Pr}^{2}-\operatorname{Pr} S\right)^{2}+\operatorname{Pr}^{2} \Omega^{2}\right]^{\frac{1}{4}},
$$$$
\alpha_{1}=\tan ^{-1}\left(\frac{\Omega}{\operatorname{Pr}}\right), \quad x_{1}=\operatorname{Pr}+\left[\left(\operatorname{Pr}^{2}-\operatorname{Pr} S\right)^{2}+\operatorname{Pr}^{2} \Omega^{2}\right]^{\frac{1}{2}} \cos \frac{\alpha_{1}}{2},
$$

$$
A_{2}=\frac{\mathrm{Sc}+\sqrt{\mathrm{Sc}^{2}+i \Omega \mathrm{Sc}}}{2}, \quad A_{4}=\frac{A_{1}^{2}}{A_{l}^{2}-\mathrm{Sc} A_{1}-\frac{i \Omega \mathrm{Sc}}{4}}
$$

$x_{5}=x_{1}^{2}-x_{2}^{2}+2 i x_{1} x_{2}$

$$
x_{6}=2 x_{1} x_{2}-\mathrm{Sc} x_{2}-\frac{\Omega \mathrm{Sc}}{4}
$$

$x_{7}=\frac{x_{1}^{2} x_{5}-x_{2}^{2} x_{5}+2 x_{1} x_{2} x_{6}}{\left(x_{5}^{2}+x_{6}^{2}\right)}$

$$
x_{8}=\frac{x_{6}\left(x_{1}^{2}-x_{2}^{2}\right)+2 x_{1} x_{2} x_{5}}{\left(x_{5}^{2}+x_{6}^{2}\right)}
$$

$A_{4}=x_{7}+i x_{8}, \quad x_{9}=1-x_{7}, \quad A_{3}=1-A_{4}, \quad x_{3}=\mathrm{Sc}+\alpha_{2}^{\frac{1}{2}} \cos \frac{\beta_{2}}{2}, \quad x_{4}=\alpha_{2}^{\frac{1}{2}} \sin \frac{\beta_{2}}{2}$

where $\alpha_{2}=\operatorname{Sc}\left(\mathrm{Sc}^{2}+\Omega^{2}\right)^{\frac{1}{2}}, \quad \beta_{2}=\tan \left(\frac{\Omega}{\mathrm{Sc}}\right), \quad a_{1}=\frac{M}{1+m^{2}}, \quad a_{2}=\frac{M m}{1+m^{2}}, \quad a_{3}=1-\alpha i \Omega$ 


$$
\begin{aligned}
& A_{5}=\Omega^{2} \alpha+a_{1}-a_{2} \Omega \alpha, \quad A_{6}=\Omega-a_{2}-a_{1} \Omega \alpha, \quad A_{8}=\frac{-\mathrm{Gr}-\mathrm{Gc} A_{4}}{4}, \\
& A_{9}=A_{1}^{2} a_{3}-A_{1}-\frac{a_{1}}{4}, \quad A_{10}=\frac{a_{2}-\Omega}{4}, \quad A_{15}=e^{-i \Omega t}-A_{11}-A_{14}, \\
& x_{15}=\frac{A_{8} A_{9}}{\left(A_{9}^{2}+A_{10}^{2}\right)}, \quad x_{16}=\frac{A_{8} A_{9}}{\left(A_{9}^{2}+A_{10}^{2}\right)}, \\
& A_{12}=A_{2}^{2}-A_{2} \frac{a_{1}}{4}, \quad A_{13}=\frac{\Omega}{4}-\frac{a_{2}}{4}, \quad A_{11}=x_{15}+i x_{16}, \\
& x_{17}=\frac{-\mathrm{Gc} A_{3} A_{12}}{\left(A_{12}^{2}+A_{13}^{2}\right)}, \quad x_{18}=\frac{i \mathrm{Gc} A_{3} A_{13}}{\left(A_{12}^{2}+A_{13}^{2}\right)}, \quad A_{15}=e^{-i \Omega t}-A_{11}-A_{14}, \\
& A_{7}=\frac{1+\sqrt{A_{5}+i A_{6}}}{2(1-i \Omega \alpha)}, \quad A_{11}=\frac{A_{8}}{A_{9}+i A_{10}}, \quad A_{14}=-\frac{\mathrm{Gc} A_{3}}{A_{12}+i A_{13}}, \\
& U(\eta, t)=e^{-x_{11} \eta}\left[\left(\cos \Omega t-x_{15}-x_{17}\right) \cos \left(\Omega t-x_{12} \eta\right)+\sin \left(\Omega t+x_{16}+x_{18}\right) \sin \left(\Omega t-x_{12} \eta\right)\right]+ \\
& +e^{-x_{1} \eta}\left[x_{15} \cos \left(\Omega t-x_{2} \eta\right)-x_{16} \sin \left(\Omega t-x_{2} \eta\right)\right]+e^{-x_{3} \eta}\left[x_{17} \cos \left(\Omega t-x_{4} \eta\right)-x_{18} \sin \left(\Omega t-x_{4} \eta\right)\right] \text {, } \\
& w(\eta, t)=e^{-x_{11} \eta}\left[-\left(\sin \Omega t+x_{16}+x_{18}\right) \cos \left(\Omega t-x_{12} \eta\right)+\sin \left(\Omega t-x_{12} \eta\right)\left(\cos \Omega t-x_{15}-x_{17}\right)\right]+ \\
& +e^{-x_{I} \eta}\left[x_{15} \sin \left(\Omega t-x_{2} \eta\right)+x_{16} \cos \left(\Omega t-x_{2} \eta\right)\right]+e^{-x_{3} \eta}\left[x_{18} \cos \left(\Omega t-x_{4} \eta\right)+x_{17} \sin \left(\Omega t-x_{4} \eta\right)\right] \text {. }
\end{aligned}
$$

If $\tau_{1}$ and $\tau_{2}$ are the axial and the transverse components of the skin-friction respectively, then

$$
\tau_{1}+i \tau_{2}=\mu\left(\frac{\partial \psi}{\partial \eta}\right)_{\eta=0}
$$

In a non-dimensional form $\tau_{l}$ becomes

$$
\tau_{l}=\left(\frac{\partial u}{\partial \eta}\right)_{\eta=0}
$$

Similarly, shearing stress at the wall along the $z$-axis is given by

$$
\tau_{2}=\left(\frac{\partial w}{\partial \eta}\right)_{\eta=0}
$$


The rate of heat transfer in terms of the Nusselt number is given by

$$
\mathrm{Nu}=\frac{q v}{V_{0} \kappa\left(T_{W}-T_{\infty}\right)}
$$

where $q=-\left.\kappa \frac{\partial T}{\partial y}\right|_{y=0}$ in a non dimensional form it is given by

$$
\mathrm{Nu}=\left(\frac{\partial \theta}{\partial \eta}\right)_{\eta=0}
$$

The rate of mass transfer is given by

$$
J^{*}=-\left.\rho D \frac{\partial C^{*}}{\partial y}\right|_{y=0} .
$$

The coefficient of mass transfer, which is generally known as the Sherwood number Sh is given by

$$
\mathrm{Sh}=\frac{J^{*} v}{V_{0} \rho D\left(C_{W}-C_{\infty}\right)}=-\left.\frac{\partial C}{\partial \eta}\right|_{\eta=0} .
$$

\section{Result and discussion}

The effect of Hall current on an MHD free convection flow of a elastico-viscous fluid past an impulsively started infinite vertical plate with mass transfer has been studied in the preceding sections. In order to get a physical insight into the problem, the velocity, temperature, concentration fields, shear stress, rate of heat and mass transfer have been discussed by assigning a numerical value to $M$ (magnetic parameter), $m$ (Hall parameter), $\alpha$ (non-Newtonian parameter), Sc (Schmidt number) and $\Omega$ (frequency parameter). The values of the Prandtl number (Pr), are taken equal to 3 and 10 which represent a saturated liquid (Freon $\mathrm{cF} 2 \mathrm{cl} 2$ ) at $272.30 \mathrm{~K}$ and gasoline $1 \mathrm{~atm}$, pressure and $200 \mathrm{C}$ respectively. The value of the Grashof number $(\mathrm{G})$ and modified Grashof number $(\mathrm{Gc})$ are taken equal to 5 and 2 respectively.

Figures 1,2 depict the variation of the velocity component $u$, for $\mathrm{G}=5.0, \mathrm{Gc}=2.0$ and $\mathrm{Pr}=3$ taking different values of $\mathrm{m}$ and $M$. It is observed that an increase in the Hall parameter $\left(\mathrm{m}=\omega_{e} \tau_{e}\right)$ leads to a rise in the velocity while a reverse effect is observed for the applied magnetic field $(M)$ for both Newtonian and non-Newtonian fluids. Figure 3 shows the variation of velocity with Sc. The velocity is greater for ammonia $(\mathrm{Sc}=0.78)$ than for helium $(\mathrm{Sc}=0.30)$. It can be seen that as $\mathrm{Sc}$ is increases velocity decreases. Figures 4 and 5 exhibit the change of behavior of the velocity component $u$ against $\eta$ under the effect of the frequency parameter, Soret number and Hall parameter respectively. An increase in frequency increases the velocity. From Fig.5 it is clear that an increase in the applied Soret number decreases the velocity. Figures 6, 7 and 8 show the velocity profile for $\operatorname{Pr}$ (Prandtl number), $S$ (heat source parameter), $\alpha$ (non-Newtonian parameter). It can be seen that velocity decreases as Pr increases whereas with an increase in $S$ velocity decreased. It is also noticed that velocity for the Newtonian fluid $(\alpha=0)$ is greater than for the non-Newtonian fluid $(\alpha \neq 0)$. 


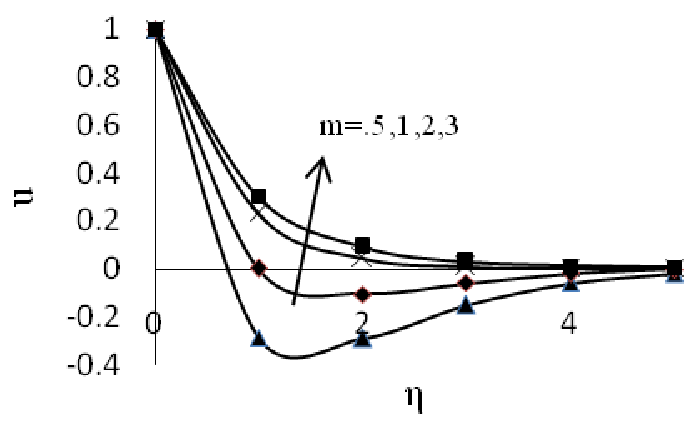

Fig.1. Effect of Hall current parameter on $u$.

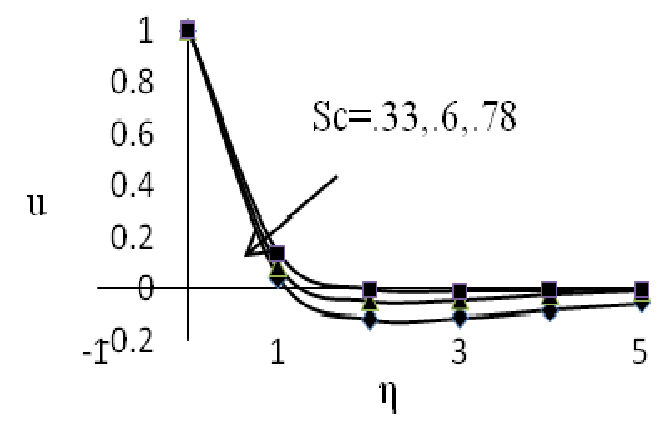

Fig.3. Effect of Schmidt number parameter on $u$.

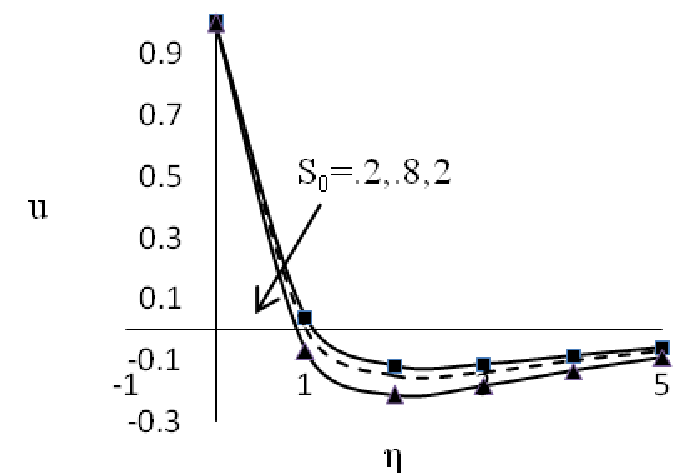

Fig.5. Effect of Soret number parameter on $u$.

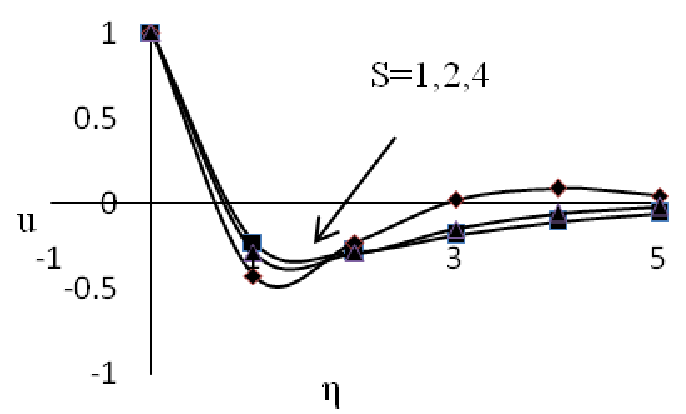

Fig.7. Effect of heat source parameter on $u$.

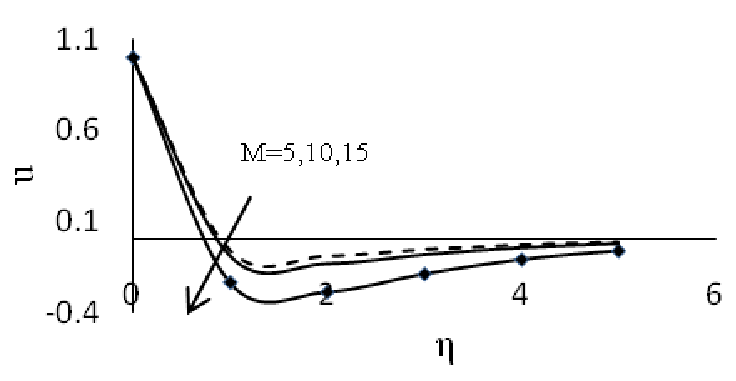

Fig.2. Effect of magnetic field parameter on $u$.

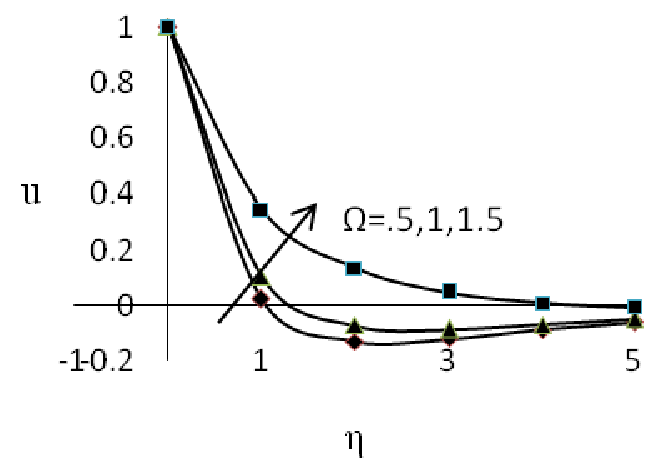

Fig.4. Effect of frequency parameter $\Omega$ on $u$.

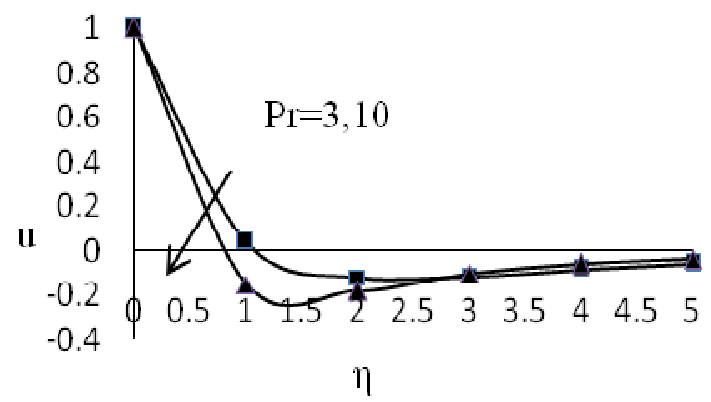

Fig.6. Effect of Prandtl number on $u$.

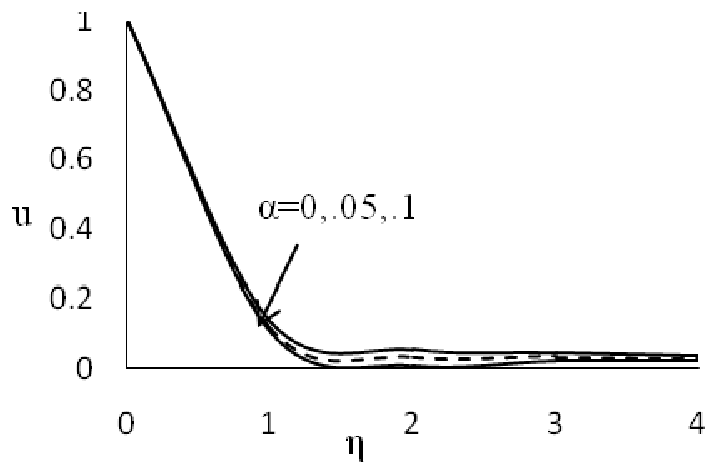

Fig.8. Effect of non-Newtonian parameter $\alpha$ on $u$. 
Figures $9,10,11,12$ represent the velocity component $w$, for $\mathrm{G}=5, \mathrm{Gc}=2$ and $\mathrm{Pr}=3$. An increase in the applied magnetic field $(M)$, Schmidt number $(\mathrm{Sc})$ and frequency $(\Omega)$ decreases the velocity in the $z$ direction for both the fluids, whereas an increase in the Hall parameter $(\mathrm{m})$ increases the velocity. Further it is noticed that $w$-component of velocity of the Newtonian fluid is lower than that of the elasticoviscous fluid. Figures 13, 14, 15 demonstrate the variation of the Soret number (So), non-Newtonian parameter $(\alpha)$ and heat source parameter $(S)$ against $\eta$. As we can see $w$ increases when So decreases. The increasing value of $\alpha$ causes $w$ decreases while velocity increases as $S$ decreases.

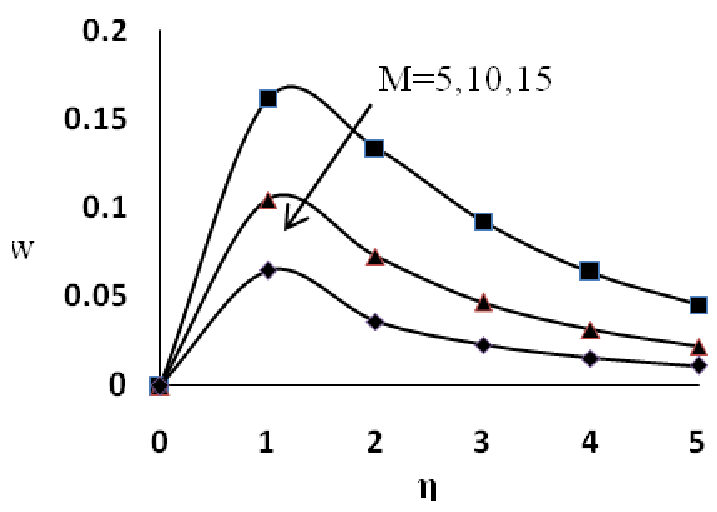

Fig.9. Effect of magnetic field parameter on velocity component $w$.

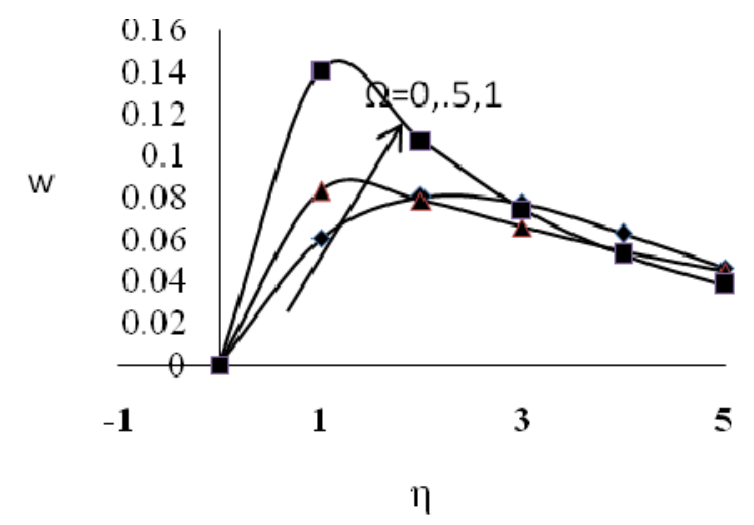

Fig.11. Effect of frequency parameter $\Omega$ on velocity component $w$.

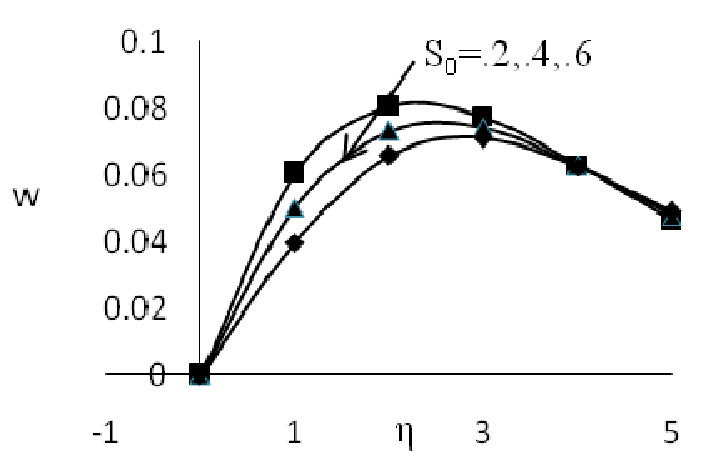

Fig.13. Effect of Soret number parameter on velocity component $w$.

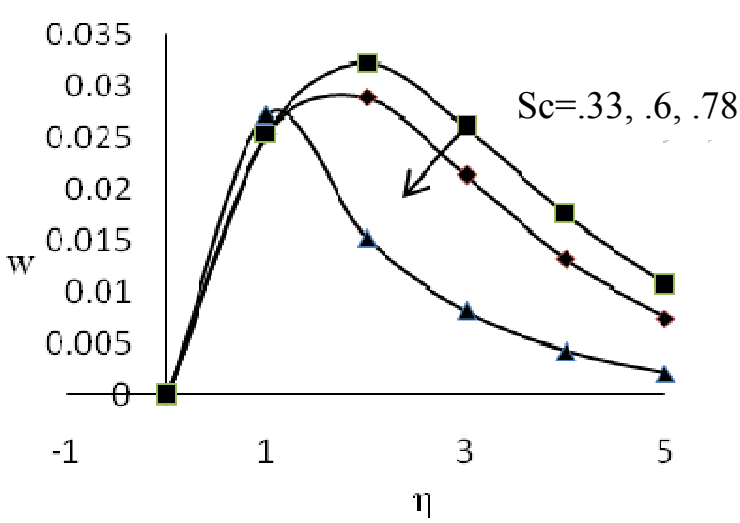

Fig.10. Effect of Schmidt number on velocity component $w$.

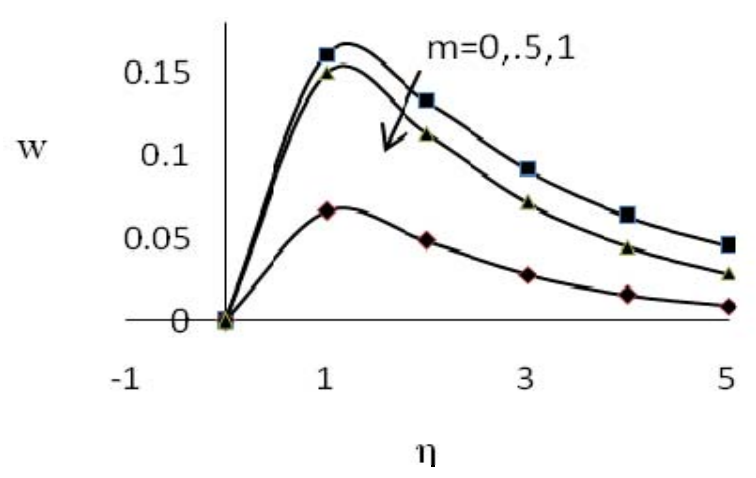

Fig.12. Effect of Hall current parameter on velocity component $w$.

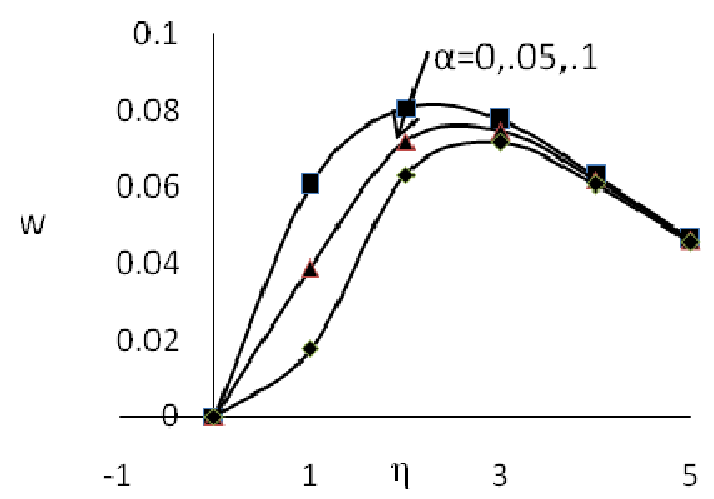

Fig.14. Effect of non-Newtonian parameter on velocity component $w$. 


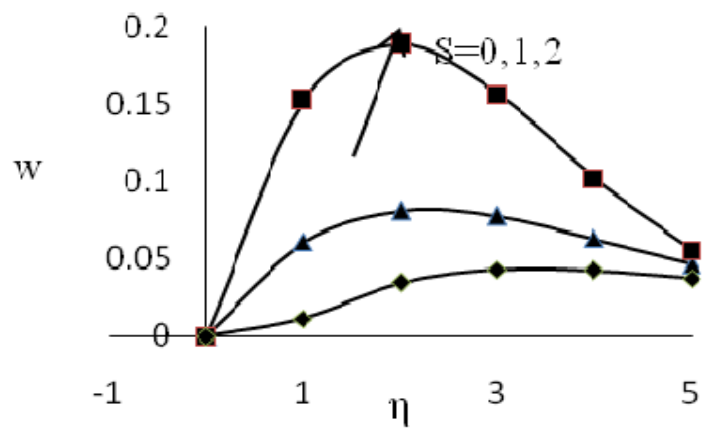

Fig.15. Effect of heat source parameter on velocity component $w$.

The temperature profiles are shown in Figs 16, 17. The effect of frequency parameter $\Omega$ and heat source parameter $S$ on temperature field $\theta$ against $\eta$ are shown, respectively. Figure16 depicts the variation in temperature. It can be seen that temperature decreases as the value of $S$ increases. The variation for $\Omega$ is shown in Fig.17. It is observed that temperature increases as $\Omega$ increases. The temperature profiles are shown in Fig. 18 for $\operatorname{Pr}=3$ and 7. The temperature distribution decreases with an increase in $\operatorname{Pr}$.
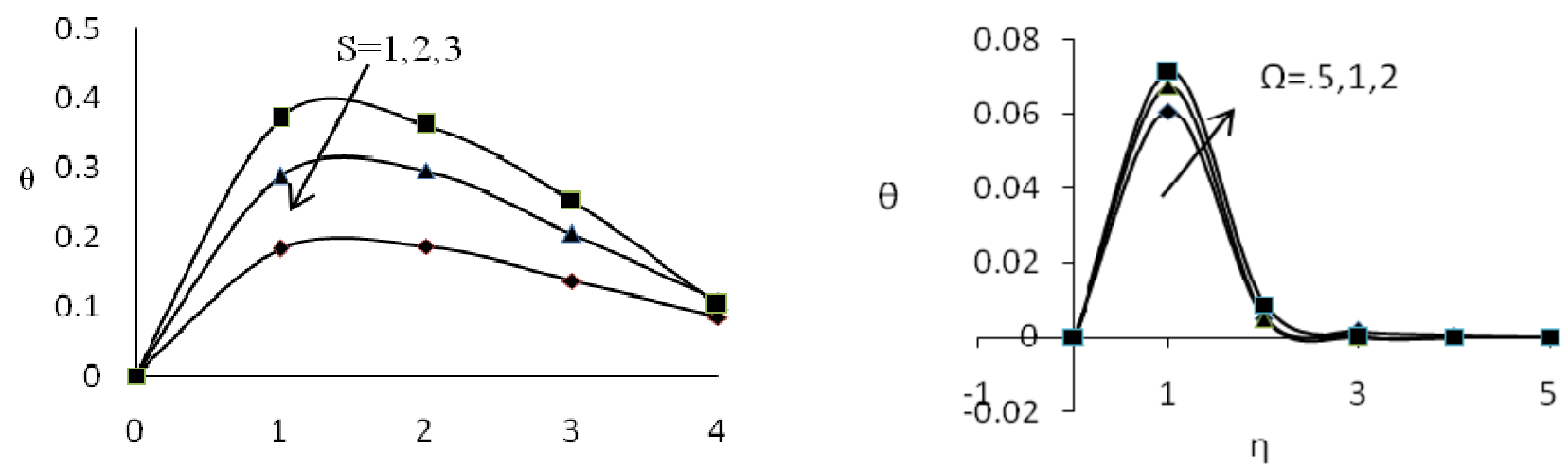

Fig.16. Effect of heat source parameter on temperature. Fig.17. Effect of frequency parameter on temperature.

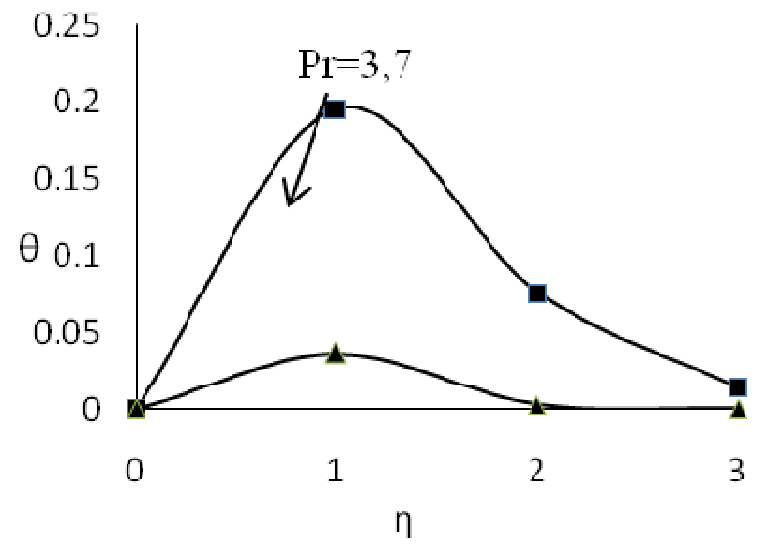

Fig.18. Effect of Prandtl number parameter on temperature.

Figure 19 presents the concentration profiles for helium $(\mathrm{Sc}=0.30)$ and ammonia $(\mathrm{Sc}=0.78)$. We noticed that increasing the value of Sc decreases the concentration profiles. This is consistent with the fact that on increase in Sc means a decrease of molecular diffusivity $D$ that results in a decrease of concentration 
boundary layer. Hence concentration of the species is higher for small values of Sc and lower for larger values of Sc. From Fig.20 it is inferred that $C$ increases as So increases whereas from Fig.21 we notice that $C$ increases sharply for a small value of $\eta$ and then again declines steadily as $\eta \rightarrow \infty$, for any value of So and $\mathrm{m}$. Further, the Soret effect on $C$ is negligible near the plate-surface and is marked at large distances from the plate-surface. A rise in so causes a greater chemical thermal diffusivity at the plate's surface. Hence, $C$ rises as So increases.

The shearing stress along the $x$-axis $\tau_{l}$ is shown in Fig. 22 for different values of So and the elastic parameter $\alpha$. It is observed that there is a rise in $\tau_{l}$ with increasing the Soret number but it falls with increasing $\alpha$.

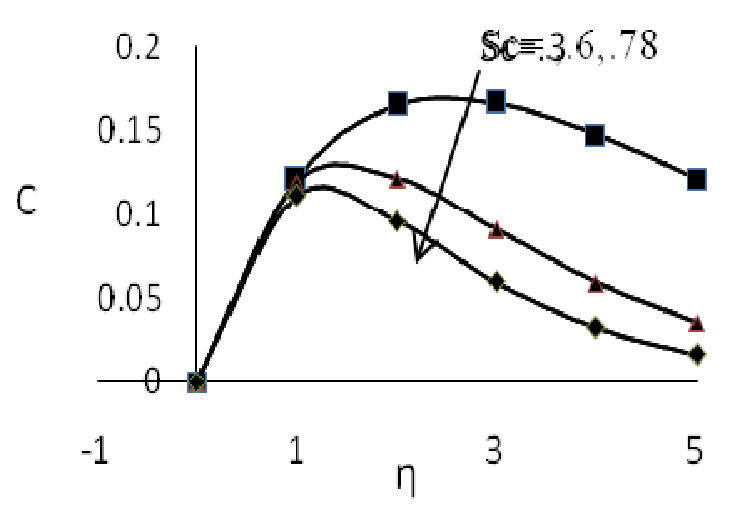

Fig.19. Effect of Schmidt number parameter on concentration.

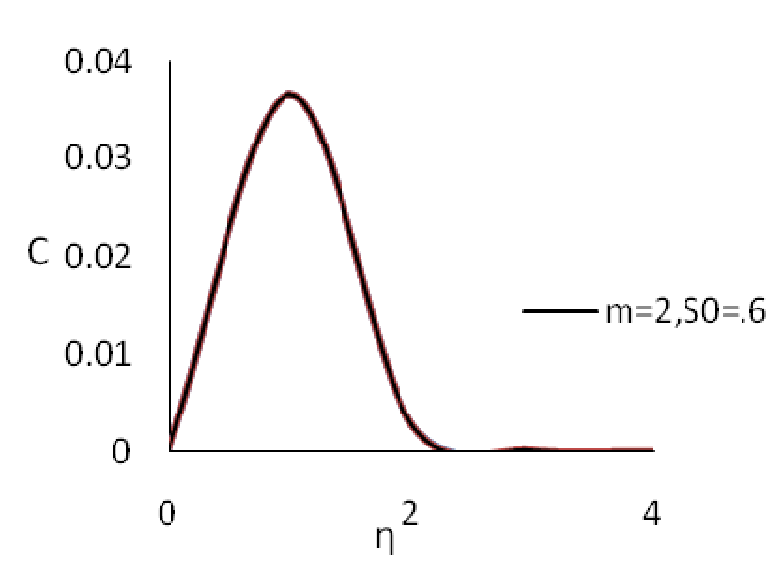

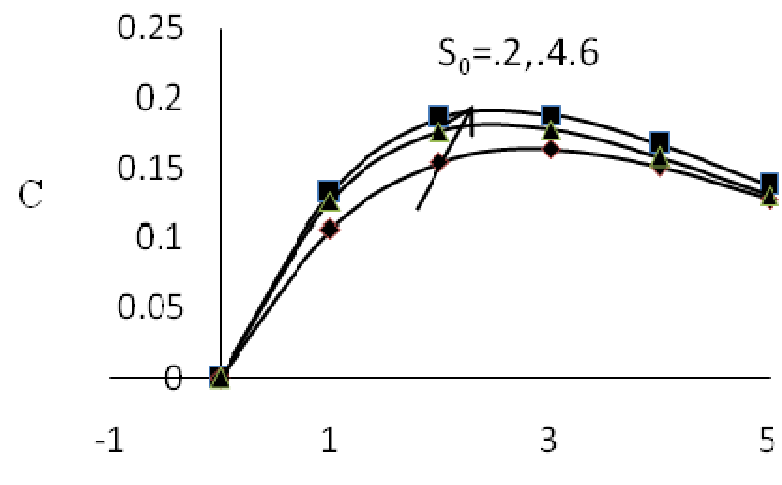

$\eta$

Fig.20. Effect of Soret number parameter on concentration.

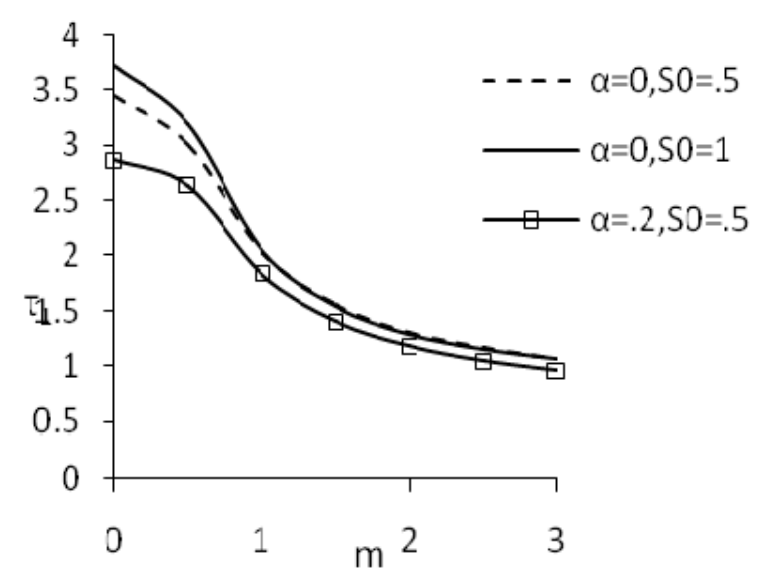

Fig.21. Effect of Hall effect number parameter on concentration. Fig.22. Variation of shearing stress $\tau_{l}$ for $S$.

Figure 23 illustrates the shearing stress along the $z$-axis $\left(\tau_{2}\right)$. From this we concluded that for the non-Newtonian fluid $\tau_{2}$ is greater than for the Newtonian fluid. However, an increase in So and $\alpha$ increases the skin friction $\tau_{2}$. Here it is also concluded that the values of $\tau_{1}$ and $\tau_{2}$ increase with the increase in the Hall parameter $(\mathrm{m})$. Likewise the effect of the heat source parameter on the Nusselt number is elucidated in Fig.24. It can be noticed that the Nusselt number noticeably decreases as $S$ increases. In Fig.25 the effect of the Soret number parameter on the Sharewood number is observed. It is noticed that Sh increases as So increases. 


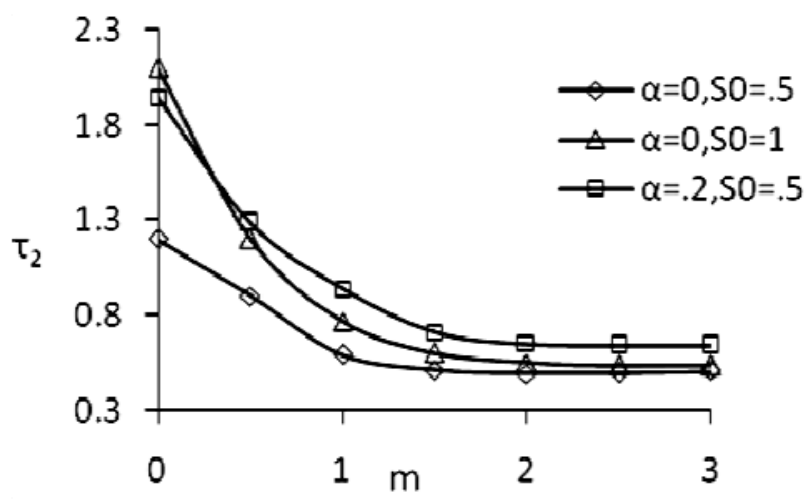

Fig.23. Variation of shearing stress $\tau_{2}$ for $S$.

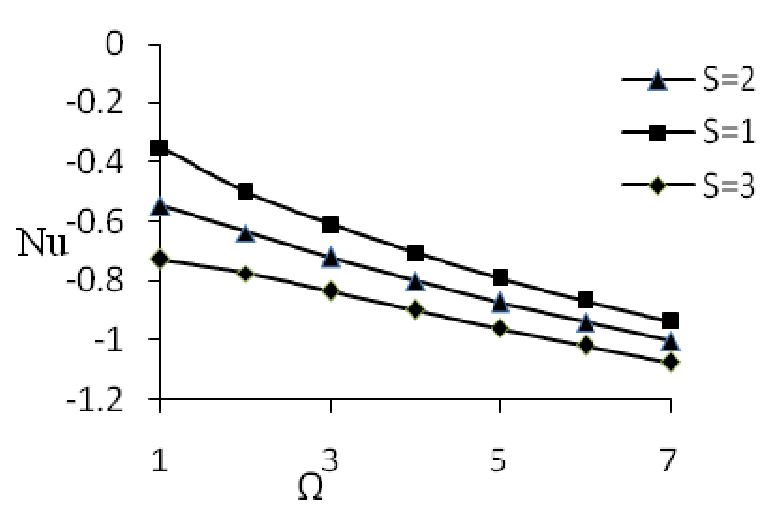

Fig.24. Variation of Nusselt number for different values of heat source parameter.

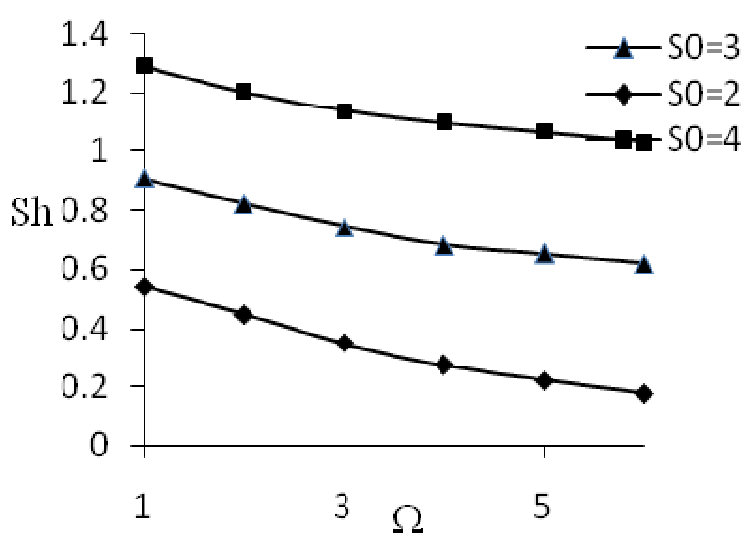

Fig.25. Variation of Sherwood number for different values of Soret number.

\section{Nomenclature}

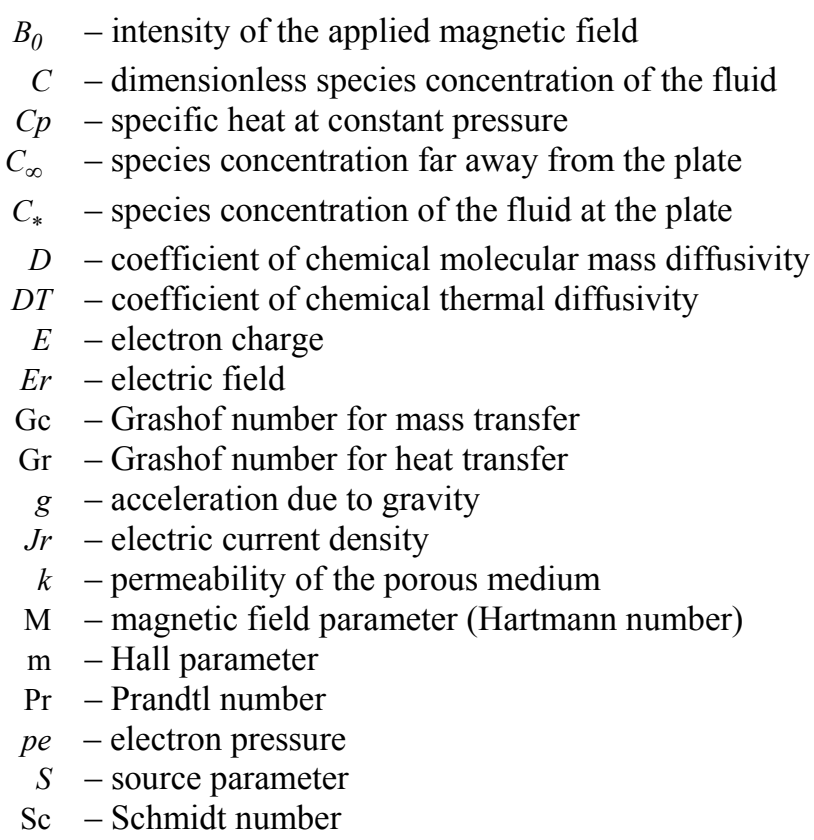




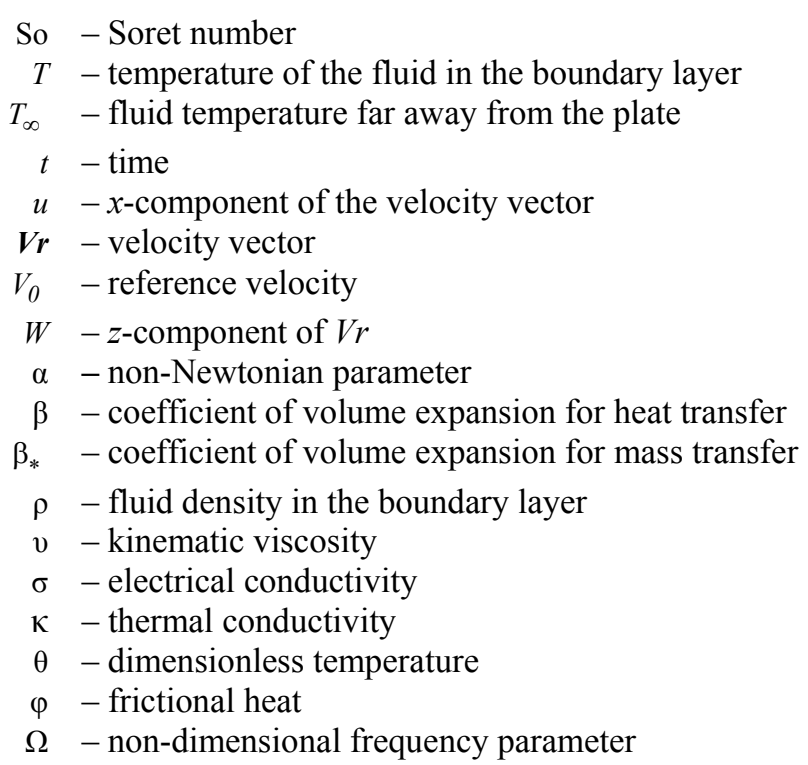

\section{References}

Aboeldahab E.M. and Elbarbay E.M.E. (2001): Hall current effect on MHD free convection flow past a semi-infinite vertical plate with mass transfer. - Int. J. Eng. Sci., vol.39, p.64.

Acharya M., Dash G.C. and Singh P.L. (2001): Hall effect simultaneous thermal and mass diffusion on unsteady hydromagnetic flow near an accelerated vertical plate. - Ind. J. Physics B, vol.75 (1), p.168.

Acharya M., Dash G.C. and Singh P.L. (2001): Effect of chemical and thermal diffusion with Hall current on unsteady hydromagnetic flow near an infinite vertical porous plate. - Journal of Physics D., Applied Physics, vol.28(12), pp.2455-64.

Alam M.S., Rahman M.M., Maleque A.M. and Ferdows M. (2006): Dofour and Soret effect on steady MHD combined free-forced convective and mass transfer flow past a semi-infinite vertical plate. - Thammsat. Int. J. Sci. Tech., vol.11, pp.1-12.

Chaudhary R.C. and Jha A.K. (2008): Heat and mass transfer in elastico-viscous fluid past an impulsively started infinite vertical plate with hall effect. - Latin American Applied Research, vol.38, p.17-26.

Chaudhary R.C. and Preeti J. (2006): Hall effect on MHD mixed convection flow of a viscoelastic fluid past an infinite vertical porous plate with mass transfer and radiation. - Theoretical Applied Mechanics, vol.33(4), p.28.

Chein H.C. (2004): Combined heat and mass transfer in MHD free convection from a vertical surface with ohmic heating and viscous dissipation. - Int. J. Eng. Sci., vol.42, p.699.

Crammer K.P. and Pai S.L. (1973): Magneto Fluid Dynamics for Engineers and Applied Physicists. - New York: Mc Graw Hill Book. Co.

Elbashbesy E.M.A. (1997): Heat and mass transfer along a vertical plate with variable surface temperature and concentration in the pressure of the magnetic field. - Int. J. Eng. Sc., vol.34, p.515.

Ferraro V.C.A and Plumption C. (1966): An Introduction to Magnetic Fluid Mechanics. - Oxford: Clarandon Press.

Gupta A.S. (1975): Hydromagnetic flow past a porous flat plate with hall effects. - Acta. Mech., vol.22, p.281.

Hossain M.A. and Rashid R.I.M.I. (1987): Hall effect on hydromagnetic free convection flow along a porous flat plate with mass transfer. - J. Phys. Soc. Japan, vol.50, p.817.

Katagiri M. (1969): The effect of Hall current on the MHD boundary layer flow past a semi-infinite flat plate. - J. Phy. Soc. Japan, vol.27, p.1051. 
Pal D. and Mondal H. (2011): Effect of Soret-Dofour chemical reaction and thermal radiation on MHD non Darcy unsteady mixed convective heat and mass transfer through over a stretching sheet. - Commun. Non-Linear Sci. Numer Simulat., vol.6, pp.1942-1958.

Pal D. and Mondal H. (2011): MHD non Darcian mixed convection heat and mass transfer over non linear stretching sheet with Soret-Dufour effect and chemical suction. - Int. Commun. Heat Mass Trans., vol.38, pp.463-467.

Pop I. and Soundalgekar V.M. (1974): Effects of hall currents on hydro-magnetic flow near a porous plate. - Acta., Mech., vol.20, p.315.

Sarpakaya T. (1961): Flow of non-Newtonian fluid in a magnetic fluid. - AICHE, J., vol.7, p.324.

Sharma et al. (2007): Hall effect on MHD convective flow of a viscous incompressible fluid past a vertical porous plate immersed in porous medium with heat source/sink. - Rom. Journ. Phys., vol.52,( 5-7), pp.487-503.

Shercliff J.A. (1965): A Text Book of Magnetohydrodynamics. - London: Pergamon Press.

Singh A.K. (2001): MHD free convection and mass transfer flow with heat source and thermal diffusion. - J. Energy Heat Mass Transfer, vol.23, p.227.

Singh A.K., Singh A.K. and Singh N.P. (2003): Heat and mass transfer in MHD flow of a viscous fluid past a vertical plate under oscillation suction velocity. - Indian J. Pure Appl. Math., vol.34, p.429.

Received: February 9, 2013

Revised: August 8, 2013 